The risk of secondary traumatic stress in the qualitative transcription process: $A$ research note

\begin{abstract}
It is recognised that transcribing is not merely a neutral and mechanical process, but is active and requires careful engagement with the qualitative data. Whether the researcher transcribes their own data or employs professional transcriptionists the process requires repeated listening to participants' personal narratives. This repetition has a cumulative effect on the transcriptionist and hearing the participants' personal narratives of a sensitive or distressing nature, can have an emotional impact. However, this potential emotional impact is often not something which is accounted for in the planning stages of research. In this paper we critically discuss the importance of considering the effects on transcriptionists who engage with qualitative data.
\end{abstract}

Key words: Transcription, trauma, emotional impact, researcher safety, qualitative 


\section{The risk of secondary traumatic stress in the qualitative transcription process: A}

\section{research note}

This article presents a brief account of literature that has considered the psychological impact of working with qualitative data. Specifically, we focus on the emotional effects that may be experienced during the process of transcription. This is particularly pertinent when working with data which is emotive such as interviews with vulnerable populations or covering topics that are painful or upsetting for the participants to discuss, which is common in qualitative research. This is especially important as some qualitative researchers actively seek to build a rapport with their participants who consequently are more likely to narrate their sensitive, emotional, and/or traumatic experiences (Liamputtong, 2007). The following research note offers a reflective discussion about the potential effects of repeated exposure to sensitive data during the transcription process.

There is a growing literature paying attention to the wellbeing of researchers during data collection. This literature has drawn attention to their physical (Ensign, 2003; Sampson et al, 2008; author and author, 2012) and emotional safety (Dickson-Swift et al., 2009). While it is acknowledged that it may be a natural response for researchers to experience a range of emotions including frustration, guilt and anger, when exposed to participants' 
experiences (Malacrida, 2007), it may be problematic when experienced at unmanageable levels, or impact on psychological functioning. This is despite the encouragement of social scientists to 'distance themselves' from the subjects of their investigation, thus ostensibly minimising the 'dramatic intensity' and 'emotional force' of the person's adverse experience (Bourdieu, 1999). However, little attention has been paid to the actual impact on researchers during transcription. The process of transcription by its nature involves the repeated listening of data to capture the words spoken and this repeated listening of disturbing material can have a lasting impact (Gregory et al., 1997). While often researchers do their own transcribing, this concern is wider than simply a researcher emotional safety issue, as many researchers/research teams employ the independent services of professional transcriptionists whose wellbeing is also important.

\section{Transcriptionist wellbeing: Outlining the argument}

Transcription was historically viewed as simply a mundane task (Lapadat and Lindsay, 1999) and has often tended to be regarded as an onerous and time-consuming activity. Thus, inexperienced researchers are often guided to plan their time-management and are trained in particular transcription notation conventions. However, as the literature 
demonstrates, consideration of the potential emotional impact of the content of the data to be transcribed is often overlooked or unrecognised as being concerning.

Our general interest in researcher wellbeing has evolved into a realisation of the importance of taking the emotional safety of both the researcher and transcriptionist into account when planning. This has been precipitated by both our own personal research and recent empirical investigations involving interviewing transcriptionists about their experiences. The combination of these empirical explorations and personal experiences has consolidated our suspicion that the process of transcription warrants more careful consideration regarding its potential for causing secondary emotional stress.

In order to make what could be a rather theoretical argument more concrete in terms of the kinds of data researchers transcribe we provide three brief examples from our own family therapy data below. This is to illustrate the sensitive and potentially distressing/shocking material that researchers and transcriptionists may be required to hear. First is a reference to the inappropriate sexual behaviour of two children. Second is a report of incest toward a child. Third is a narrative of physical violence toward a child.

Webber family: 
Mum:

he can't go to the toilet on his own 'cause he's there I say you know Stuart's now told us that you know he's sucked his willy

FT: Right

Mum: you know that is not a child thing you don't do that as a kid

\section{Webber family}

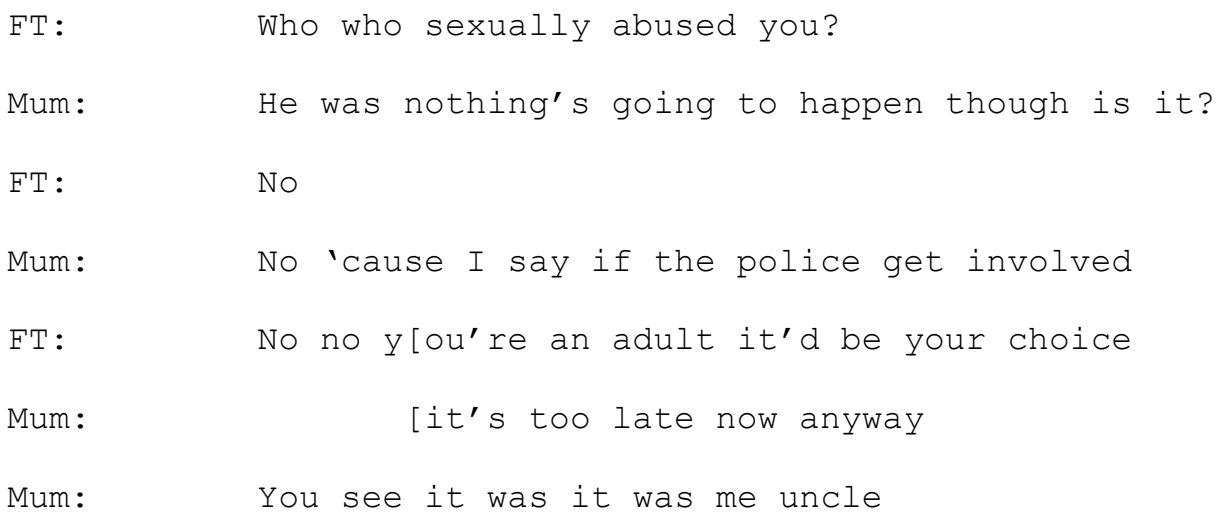

\section{Clamp family}

Dad:

and he was bangin' 'is 'ead against the wall

punching 'im in the face and everythin' when I told

'im to leave 'im alone, 'e told me no

FT: $\quad$ Hum

Dad so I said right fair enough you've gone too far now so I took me belt off and smacked 'is bum twice 


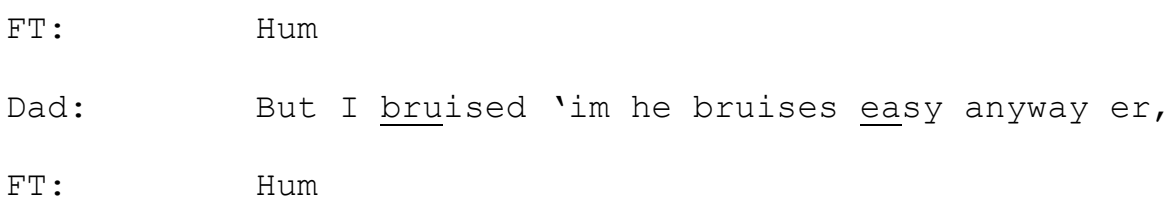

We have provided these examples to illustrate the types of issues that may arise when collecting qualitative data. In terms of family therapy, the qualitative researcher may anticipate the possibility of sensitive issues being raised and may be prepared. However, these may be unexpected if the researcher and/or transcriptionist was not present during data collection. In the case of this corpus, the data were naturally-occurring and the researcher was not present during the therapeutic session, but was given the videorecordings afterwards. Thus we argue that there was a greater likelihood of unanticipated emotional impact. However, even in the case of interview or focus group research whereby researchers are aware that the topic is sensitive and they have a research schedule to follow, it is possible that unanticipated responses may arise. Furthermore even when anticipated, actually hearing the voices of participants (who may be in some circumstances either victims or perpetrators) can be distressing/shocking for the researcher/transcriptionist. This is compounded further through repeated listening; especially given that actually hearing participants' voices is likely to trigger a greater emotional reaction than simply reading written data (Shopes, 2013). Furthermore, while it is standard practice for transcriptionists to transcribe audiodata, when transcribing video (although there is no empirical evidence), it could be 
hypothesised that this is even more stressful as the distress of the participants is more visible.

\section{The emotional reactions precipitated by transcribing distressing data}

As Bahn and Wetherill (2012) point out, the possibility of either physical or emotional harm to researchers needs to be adequately risk-assessed and managed by research teams. This should include any professional transcriptionists employed on the project. Evidence for this has been indicated in a recent study of transcriptionists in Australia and New Zealand whereby some reported being so overwhelmed emotionally that they made the active decision to refuse further potentially emotional transcription work (Wilkes et al., 2014). After publishing a paper on the physical safety risks inherent in qualitative data collection we became aware that the transcriptionist was emotionally affected by the data (see author and author, 2012). This provoked us to extend our investigation to explore in more detail the potential impact of listening to troubling data on transcriptionists. We provide two short extracts below from the original paper (author and author, 2012) to illustrate the nature of the emotions experienced by the transcriptionist that prompted our further interest.

\footnotetext{
"I can't say it frightened me but I felt I felt that it was a must have been en- horrendous nightmarish situation for you to be in. It is
} 
the stuff of nightmares isn't it really cos how do you get out of that house?"

"There is a feeling of, that you can't do anything about it"

While the nature of the topic being researched for this project was not overtly sensitive, the feelings of concern and helplessness experienced by the transcriptionist were sufficient to warrant debriefing. Thus, we followed this theme of research by conducting interviews with a further 8 people involved in transcribing qualitative data. We were surprised to find a consistency across the sample in terms of how emotionally affected these individuals were, with emergent themes of secondary traumatic stress and vicarious traumatisation.

\section{The possibility of transcriptionists' secondary stress and vicarious traumatisation}

Secondary traumatic stress has been defined as being a 'natural consequence' of empathy between two individuals where one individual had been initially traumatised and the other is affected by hearing about those experiences (Devilley et al., 2009). Similarly vicarious traumatisation is experienced through the process of empathically engaging with other individuals' trauma narratives (Pearlman and Saakvitne, 1995). It is experienced as a form of 'emotional residue of exposure', resulting from listening to 
other people's trauma narratives and witnessing their pain and fear (American Counseling Association, fact sheet \#9).

As we found in our research, other scholars have also reported researcher's feelings of helplessness resulting from exposure to stories of emotional and traumatic events (Etherington, 2007). This emotional impact therefore is not only common for researchers collecting primary data, but may also occur for those engaged in secondary analysis (Fincham et al., 2008) whereby researchers may endure 'pain-by-proxy' (Moran-Ellis, 1997). Additionally as noted, transcriptionists can also be profoundly affected and may need to be debriefed (Etherington, 2007; Gregory et al., 1997). This may be more challenging if the transcriptionist is not directly involved in the research project because of the ethic of confidentiality. Clearly transcriptionists may become emotional when listening to sensitive data (Lalor et al., 2006) as it is difficult to stay detached when dealing with emotionally charged and personal narratives (DicksonSwift et al., 2009). Furthermore repeated exposure to the data and topic may feel overwhelming (Campbell, 2002) and this repeated exposure risks causing a cumulative effect (Coles and Mudlay, 2010).

\section{Managing emotional consequences of transcribing qualitative data}


Evidently there is to some extent a level of 'risk' of emotional distress to transcriptionists, whether it is the researcher or professional. From a broader perspective, contemporary Western society has become increasingly concerned with the possible risks of harm (Beck, 1992). However, there are differences between societal and institutional risks that are important for the research community to acknowledge. As noted by Rothstein et al. (2006) this 'colonisation' of societal concerns in institutional contexts has limitations because of the 'legitimacy of rules and methods of regulation'. In other words, the boundaries and rules of regulation can in itself create risk, or lead to a greater level of risk aversion. This culture of risk avoidance has translated to the research community (Pronovost and Sexton, 2005) as researchers have been under increasing pressure to consider, predict and manage potential adverse events (Shaw and Barrett, 2006). Although the emphasis on risk management has historically focused on participants, there is also now a greater emphasis on managing wellbeing within research teams (Dickson-Swift et al., 2008) and for researchers to care for themselves (Corbin and Morse, 2003).

Although there are guidelines regarding how to conduct qualitative research the issue of institutional risk management has received less attention (Bloor et al., 2007) especially in relation to the wellbeing of the researcher, supervisor, and transcriptionist (McCosker et al., 2001). Unfortunately transcriptionists are often assumed to be unaffected by the 
process (Gregory et al., 1997) and yet are not immune to the profoundness of the stories they hear. However, they are rarely given the chance to talk about the emotional impact experienced (Warr, 2004). In other settings such as clinical practice, emotional boundaries are clearly demarcated and emotional reflexivity is inherent in the work, but in research contexts less attention has been paid to managing the emotional impact (Dickson-Swift et al., 2006). It has however been recognised that the emotional risk is higher for researchers and transcriptionists if they have been inadequately prepared for the possible effects or if they are denied an opportunity to debrief (Etherington, 2007). Managing transcriptionists' emotional responses can be therefore integrated into qualitative research practice by ensuring both briefing and debriefing is carried out effectively within the boundaries of confidentiality.

\section{Discussion}

We have raised the issue of the potential emotional impact on those who transcribe qualitative data and transcriptionist safety should not be overlooked. This issue however has tended to be marginalised (Tilley and Powick, 2002), resulting in transcriptionists feeling unsupported and isolated (Johnson and McLeod, 2003). The limited empirical work conducted in this area has illustrated that transcriptionists feel invisible and peripheral to the research process (Gregory et al., 1997). 
We argue that this invisibility and marginalisation of transcriptionists increases the potential for emotional harm and possibly vicarious traumatisation, which has generally been unaddressed as an issue in qualitative research. Where the potential for secondary traumatic stress and emotional burnout is possible, a lack of awareness can increase individuals' vulnerability to the effects of stressful conditions (Newell and MacNeil, 2010). This increased risk is exacerbated by a naivety regarding the potential need for transcriptionists to be briefed and debriefed when handling sensitive or distressing data. While we recognise that some members of the research community do already engage and involve the transcriptionist, this is not recognised and promoted as standard practice and there are limited guidelines to support the process or guide research teams.

This poses a question regarding who is responsible (and when) for the emotional wellbeing transcription. We propose three phases whereby this becomes pertinent. First, is prior to the transcription, before the recordings are handed to the individual undertaking the task (pre). Second is during the transcription process (peri). Third is once the transcription is complete (post). While some responsibility may be assumed as routine (a brief pre-transcription assessment and short post debrief), others may only be necessary should emotional wellbeing be illuminated as concerning. 
It could be argued that the ethics committee play a role in protecting research team safety as well as participants. Currently however ethics committees' main function is to anticipate and minimise potential risk, for the protection of participants (McCosker et al., 2001). While there is a consideration of researcher safety, it is not their primary function (Bloor et al., 2007) and neither are we suggesting that it should be, but can serve as a useful prompt for researchers to reflect on the issue. Arguably research communities have an obligation in managing possible risk to transcriptionists in qualitative research. Researchers could be more proactive in publishing/reporting their experiences and raising the profile of researcher/transcriptionist wellbeing by creating a culture of sharing and transparency (Author and Author, 2012). Similarly research teams have some responsibility for risk-management by anticipating potential emotional harm in the transcription process. For example, initially reviewing the data for potentially distressing material contained within it (pre transcription). We recognise that it is possible that researchers who are familiar with handling particular kinds of data frequently may become desensitised to its emotional impact and therefore be less cognizant of its potential impact on others. It is unusual for individual researchers to be working in complete isolation, without the involvement of collaborators, coinvestigators, supervisors or other members of a research team. Thus a team approach can be effective in supporting its members especially through collaboration, participation and team cohesion (Lemieux-Charles and McGuire, 2006). Arguably 
transcriptionists also have some responsibility during the process if they begin to feel emotionally uncomfortable or if the material has a personal resonance that was unexpected. Ultimately in such cases there may be a need for the transcriptionist to withdraw from their commitment (this could be offered as an option in the pre-stage as routine).

In conclusion, we have proposed that as a qualitative research community there is an onus on us as ethical practitioners to be more aware of the possible effects of transcribing qualitative data and to take measures to manage this. We suggest considering more carefully the role of the transcriptionist regarding the potential emotional impact, and foster a culture of inclusivity. In relation to confidentiality, this is less problematic if the transcriptionist is also a researcher. However, regardless of the role within the research team, measures need to be implemented to promote briefing and debriefing in order to mitigate against possible vicarious impact of listening to sensitive or traumatic data.

\section{References}

American Counseling Association, fact sheet \#9 As retrieved on $31^{\text {st }}$ October 2014 from 
http://www.counseling.org/docs/trauma-disaster/fact-sheet-9---vicarioustrauma.pdf?sfvrsn=2

Bahn S and Weatherill P (2013) Qualitative social research: A risky business when it comes to collecting 'sensitive' data. Qualitative Research, 13 (1): 19-35. Beck U (1992) Risk Society. London: Sage

Bloor M Fincham B and Sampson H (2007) QUALITI (NCRM) Commissioned inquiry into the risk and well-being of researchers in qualitative research. Cardiff: ESRC.

Bourdieu, P. (1999). The weight of the world. Cambridge: Polity.

Campbell R (2002) Emotionally Involved: The Impact of Researching Rape. New York: Routledge.

Coles J and Mudlay N (2010) Staying safe: strategies for qualitative child abuse researchers. Child Abuse Review, 19: 56-69.

Corbin J and Morse J (2003) The unstructured interactive interview: Issues of reciprocity and risks when dealing with sensitive topics. Qualitative Inquiry. 9(3): $335-354$

Devilly GJ Wright R and Varker T (2009) Vicarious trauma, secondary traumatic stress or simply burnout? Effect of trauma therapy on mental health professionals. Australasian Psychiatry, 43(4): 373-385.

Dickson-Swift V James E Kippen S and Liamputtong P (2009) Researching 
sensitive topics: qualitative research as emotion work. Qualitative Research, 9 (1): $61-79$

Dickson-Swift V James E Kippen S and Liamputtong P (2008) Risk to researchers in qualitative research on sensitive topics: issues and strategies. Qualitative Health Research, 18 (1): 133-144.

Dickson-Swift V James E Kippen S and Liamputtong P (2006) Blurring boundaries in qualitative health research on sensitive topics. Qualitative Health Research, 16 (6): 853-871.

Ensign J (2003) Ethical issues in qualitative health research with homeless youths. Journal of Advanced Nursing, 43 (1): 43-50.

Etherington K (2007) Working with traumatic stories: from transcriber to witness. International Journal of Social Research Methodology, 10 (2): 85-97.

Fincham B Scourfield J and Langer S (2008) The impact of working with disturbing secondary data: reading suicide files in a coroner's office. Qualitative Health Research, 18 (6): 853-862.

Gregory D Russell C and Phillips L (1997) Beyond textual perfection: Transcribers as vulnerable persons. Qualitative Health Research, 7 (2): 294-300.

Johnson B and Macleod Clark J (2003) Collecting sensitive data: The impact on researchers. Qualitative Health Research, 13 (3): 421-434.

Lalor J Begley C and Devane D (2006) Exploring painful experiences: impact of 
emotional narratives on members of a qualitative research team. Journal of Advanced Nursing, 56 (6): 607-616.

Lapadat J and Lindsay A (1999) Transcription in research and practice: from standardization of technique to interpretive positioning. Qualitative Inquiry. 5 (1): 64-86.

Lemieux-Charles L and McGuire W (2006) What do we know about health care team effectiveness? A review of the literature. Medical Care Research and Review, 63: 263-300.

Liamputtong P (2007) Researching the Vulnerable. London: SAGE

Malacrida C (2007) Reflexive journaling on emotional research topics: Ethical issues for team researchers. Qualitative Health Research, 17 (10): 1329-1339.

McCosker H Barnard A and Gerber R (2001) Undertaking sensitive research: Issues and strategies for meeting the safety needs of all participants. Forum: Qualitative Social Research, 2 (1): Online.

Moran-Ellis J (1997) Close to home: The experience of researching child sexual abuse. In: M Hester L Kelly and J Radford (Eds) Women, violence and Male Power: Feminist Activism, Research and Practice. Buckingham: Open University Press.

Newell J M and MacNeil GA (2010) Professional Burnout, Vicarious Trauma, 
Secondary Traumatic Stress, and Compassion Fatigue. Best Practices in Mental Health, 6(2): 57-68.

Author and Author (2012)

Pearlman L and Saakvitne K (1995) Trauma and the Therapist: Countertransference and Vicarious Traumatisation in Psychotherapy with Incest survivors. London: Norton

Pronovost P and Sexton B (2005) Assessing safety culture: Guidelines and recommendations. Quality and Safety in Health Care 14: 231-

Rothstein, H., Huber, M., \& Gaskell, G. (2006). A theory of risk colonization: The spiralling regulatory logics of societal and institutional risk. Economy and society, 35(1), 91-112.

Sampson H Bloor M and Fincham B (2008) A price worth paying? Considering the 'cost' of reflexive research methods and the influence of feminist ways of 'doing'. Sociology, 42 (5): 919-933.

Shaw S and Barrett G (2006) Research governance: regulating risk and reducing harm? Journal of the Royal Society of Medicine, 99: 14-19.

Shopes L (2013) Oral history. In NK Denzin and YS Lincoln (Eds.) Collecting and interpreting qualitative materials (pp. 119-150). Thousand Oaks, CA: Sage. Tilley S and Powick K (2002) Distanced data: Transcribing other people's research tapes. Canadian Journal of Education, 27 (2\&3), 291-310. 
Warr D (2004) Stories in the flesh and voices in the head: reflections on the context and impact of research with disadvantaged populations. Qualitative Health Research, 14 (4), 578-587.

Wilkes L Cummings J and Haigh C (2014) Transcriptionist saturation: Knowing too much about sensitive health and social data. Journal of Advanced Nursing, DOI: $10.111 /$ jan. 12510 